\title{
Different methods to measure canine rotation
}

Neam F Agha

BDS, MSc (Lect)

Neam R Al-Saleem BDS, MD (Assist Lect)

\author{
Dept of Pedod, Orthod and Prev Dent \\ College of Dentistry, University of Mosul
}

\begin{abstract}
Aim: Demonstration of a new method which is liner measurement to record the amount of canine rotation after orthodontic treatment, in addition to the ordinary angular method for measuring canine rotation. Materials and Methods: Forty five patients (20 males and 25 females) were selected: Those need extraction of the first premolars for correction of malocclusion. Their age varied from 12-23 years. A study model was taken for them pre and post treatment, then measurements were done directly on the cast and other measurements indirectly converted on a paper to measure other variables. Results: There were no significant differences among age groups and between the sexes for the angular and linear methods. Also, there was insignificant canine rotation in the mesial or distal sides toward or away from the reference line within the different groups; this clearly seen in the linear method. In the angular method, also canine rotation can be seen within the age groups but not demonstrated the side of rotation like the linear method. Conclusion: Canine rotation occur within the treatment work in the two methods of measuring canine rotation, but this research proved that the linear direct measurement which is the new method followed in this work was more beneficial and accurate for clinical work than the indirect angular measurements.
\end{abstract}

Key Words: Canine rotation, canine retraction.

Agha NF, Al-Saleem NR. Different methods to measure canine rotation. Al-Rafidain Dent J. 2006; 6(2): 122-129.

Received: 31/10/2005

Sent to Referees: $2 / 11 / 2005$

Accepted for Publication: 18/12/2005

\section{INTRODUCTION}

Orthodontic tooth movements are based on the ability of the bone to react to mechanical stresses with apposition and resorption of the alveolar bone. So, bone remodeling laws have been used to simulate the movement of single tooth. The regular rate of osteogenesis during canine retraction is about $1 \mathrm{~mm}$ per month, which is much slower than in destruction osteogenesis. Currently, the underlying biomechanical, biophysical and cellular processes are the subject of numerous studies. ${ }^{(1-3)}$

After more than half century of research on orthodontic tooth movement, it is disappointing to conclude that the answer to the question of the optimal force is still far away. ${ }^{(4)}$

The canines share a very important role in oral functions and esthetics. Their unique position connects anterior and posterior segments of the dental arch and makes their orthodontic movement of great clinical importance, especially in premolar extraction cases, which is a very common orthodontic procedure. ${ }^{(5)}$ Therefore, the retraction of canines represents a fundament- al stage in considerable number of orthodontic movements. Correct positioning of the canine after retraction, recognized to be the upper most importance for function, stability and esthetic, can be obtained either by up-righting after uncontrolled tipping (frictional system sliding mechanism) or by mean of biomechanically predetermined and controlled movement (non frictional system closing loops). ${ }^{(6)}$

During the whole distal translation of canines, the stress in the alveolar bone is higher than that in periodontal ligaments with the cervical region affected more by the stress as that in the apical region of the alveolar bone and periodontal ligaments. ${ }^{(7,8)}$

Because the force application is at the bracket (crown of tooth) level and not through the center of resistance of the tooth in the labiolingual direction, unaccounted axial tooth rotation will occur, and a moment is necessary to counteract tooth rotation. The moment is exerted by ligature tying the arch wire to the bracket. So, rotation depends on the quality of the ligation technique to keep the arch wire seated in 
the bottom of the slot. Ligation force necessary to seat an arch wire increases as the stiffness of the arch wire increase. ${ }^{(9-12)} \mathrm{A}$ decrease in the overall bracket size and shortening of the slot length invite unaccounted tooth rotation especially with those brackets giving the arch wire increased mobility due to their shaping or lack of ligature wire. ${ }^{(13,}{ }^{14)}$ However, because of the risk of friction, the ligature tie can not be very tight. Also the ligature will probably yield during control intervals resulting in rotation. ${ }^{(12)}$

Steel ligature produces lower friction than elastomeric nodules on average but the variation was considerable, probably because of inability to standardize the tightness of the tie. ${ }^{(15)}$

The problem of rotational control during the retraction has been relieved by Jessing loop technique (frictionless retraction of the canine) ${ }^{(6)}$ The anti-rotation moment to force ratio has therefore been increased from $4: 1$ in sliding technique to $7: 1$ in the new version of the spring (Jessing loop). ${ }^{(9)}$

The aim of this research was determine the amount of canine rotation after orthodontic treatment. A new method is created to determine canine rotation, which is directly measured on study model other than the ordinary angular method, which is indirect measurements on a paper.

\section{MATERIALS AND METHODS}

Forty-five subjects (20 males and 25 females) were included in this work. The sample was obtained from patients attending for orthodontic treatment at College of Dentistry, University of Mosul and from private orthodontic clinics. The criteria of the sample were:

-Patients $12-23$ years old at start of treatment.

-Orthodontic cases planned for treatment by first premolar extraction in each quadrant (no other extraction).

-The research took maxillary arch only.

-Edge wise appliance (standard prescript-

ion) stainless steel brackets $0.022 \times$ 0.030 inch.

The samples were divided into 4 groups, two years differences for each group, which is the longest period for treating the patient from bonding till finishing the case and debonding the brackets. Those were:
The first group (12 cases) 12-14 year; the second group (12 cases) 15-17 year; the third group (11 cases) 18-20 year; and the fourth group (10 cases) 21-23 year.

\section{Treatment Method}

All the patients were treated identically: Extraction of two first premolars (right and left), then alignment and leveling of the dental arch. After this step, impression was taken for the patient then poured to obtain pre study model. This was done in order to avoid the rotated canine. Upper 0.018 inch stainless steel arch wire was placed for initial retraction of the canine, then completion of space closure by $0.016 \times$ 0.022 inch stainless steel continuous wire. The sliding mechanic done by using power chain (Kunststoff Transparent Chain) changed every 3 weeks, ${ }^{(16)}$ then en mass retraction of anterior teeth by $0.016 \times 0.022$ inch stainless steel wire which contain tear drop loop activated $1 \mathrm{~mm}$ every 21 days. After finishing the retraction, another impression was taken for the patient in order to get a post treatment study model. So, two models for each case, pre and post casts, were obtained.

The measurements were divided into two steps: The first step (direct measurements) (Figures 1 and 2) was done on the study model for each case. Pre and post treatment measurements were done by drawing reference line, which was represented by median palatal raphe, then measuring the distance from the mesial and distal sides of the canine at contact point vertical to the reference line by using vernia. Also, measuring the mesiodistal width of the canine. These measurements recorded on a paper.

Regarding the second (indirect measurements) (Figure 3), the recorded measurements were done from the study cast to the paper by drawing vertical line on the paper then the mesial and distal distances drawn vertical from the reference line. The width of the canine was drawn by the distance between mesial and distal lines. So, four linear measurements mesial and distal pre and post treatment were obtained. The angular measurement was done by drawing line continuation to canine width to meet the reference line forming canine angle. 


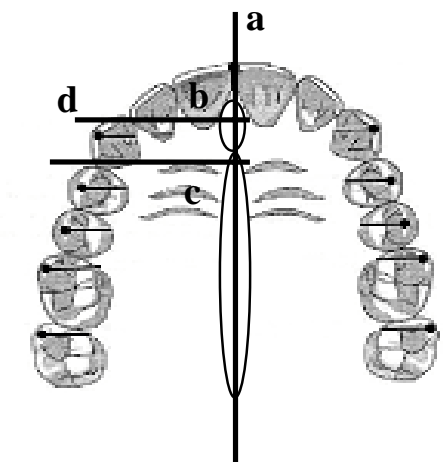

Figure (1): Pre measurements of canine distances a: Reference line. b: Mesial distance. c: Distal distance. d: Canine width.

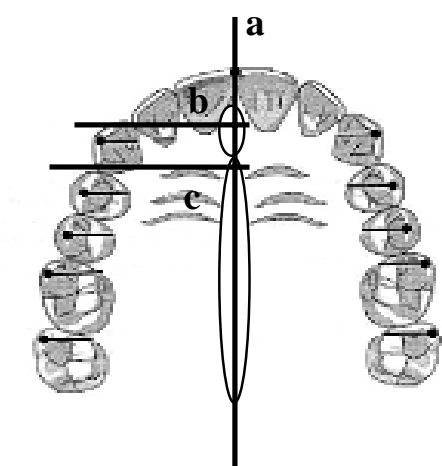

Figure (1): Post measurements of canine distances

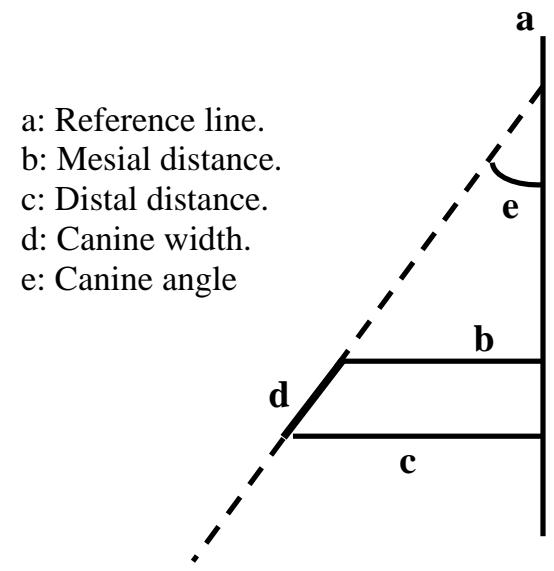

For the statistical analysis, systematic differences between males and females for each side, pre and post treatment measurements, were analyzed by Student's t-test. Also, description of the variables by using Minitab computerized statistical analysis were done at $p \leq 0.05$ level.

\section{RESULTS AND DISCUSSION}

\section{Linear Measurement-Direct Method}

Table (1) demonstrated the comparison between pre and post treatment canine position. This variable was obtained from the linear measurements: Mesial plus distal distances divided by two [(M+D)/2].
Figure (3): Indirect measurements

This gave the central canine distance from the reference line. A non- significant difference was found between the two positions that's why the effect of canine position from the reference line pre and post treatment was excluded.

Tables (2) and (3) demonstrated the comparison between males-females pre and post treatment measurements, respectively, for each side. A non-significant difference between the two genders for both tables was noticed. So, the data of the females were added to the males and analyzed together as a total.

Table (1): Comparison between pre and post treatment for canine position

\begin{tabular}{cccccc}
\hline Treatment & Mean* $^{*}$ & \pm SD & Minimum & Maximum & $\boldsymbol{p}$-value \\
\hline Pre & 15.75 & 1.53 & 11.4 & 12.5 & 0.23 \\
Post & 16.18 & 1.65 & 12.8 & 17.5 & NS \\
\hline
\end{tabular}

Number of the sample $=45$.

*Measurements in mm

SD: Standard deviation, NS: Not significant $(p>0.05)$. 
Table (2): Comparison between males-females for pre treatment measurements

\begin{tabular}{cccccccc}
\hline \multicolumn{2}{c}{ Sides } & Sex & Mean* & \pm SD & Minimum & Maximum & $\boldsymbol{p}$-value \\
\hline \multirow{4}{*}{ Mesial } & \multirow{2}{*}{ Right } & Male & 13.90 & 1.04 & 11.7 & 16.5 & 0.85 \\
& \multirow{2}{*}{ Left } & Female & 13.72 & 1.54 & 12.5 & 21.9 & NS \\
& & Male & 13.70 & 0.83 & 11.0 & 15.7 & 0.62 \\
& & Female & 14.20 & 1.70 & 12.0 & 21.0 & NS \\
\hline \multirow{4}{*}{ Distal } & \multirow{2}{*}{ Right } & Male & 18.73 & 1.20 & 11.1 & 17.6 & 0.86 \\
& \multirow{2}{*}{ Left } & Female & 18.52 & 1.50 & 10.7 & 22.5 & NS \\
& & Male & 18.66 & 1.11 & 12.2 & 17.5 & 0.91 \\
& & Female & 18.70 & 0.99 & 11.9 & 21.9 & NS \\
\hline
\end{tabular}

*Measurements in m; Number of the sample: 20 males and 25 females.

SD: Standard deviation, NS: Not significant $(p>0.05)$.

Table (3): Comparison between males -females for post treatment measurements

\begin{tabular}{lccccccc}
\hline \multicolumn{2}{c}{ Sides } & Sex & Mean* & \pm SD & Minimum & Maximum & $\boldsymbol{p}$-value \\
\hline \multirow{4}{*}{ Mesial } & \multirow{2}{*}{ Right } & Male & 14.50 & 1.20 & 12.4 & 19.0 & 0.41 \\
& & Female & 13.80 & 0.89 & 14.8 & 22.0 & NS \\
& \multirow{2}{*}{ Left } & Male & 14.48 & 1.10 & 11.9 & 18.7 & 0.32 \\
& & Female & 14.00 & 1.00 & 13.7 & 21.5 & NS \\
\hline \multirow{4}{*}{ Distal } & \multirow{2}{*}{ Right } & Male & 18.80 & 0.98 & 12.0 & 19.5 & 0.21 \\
& \multirow{2}{*}{ Left } & Female & 18.35 & 1.20 & 13.5 & 21.5 & NS \\
& & Male & 18.81 & 0.99 & 11.6 & 25.7 & 0.11 \\
& & Female & 18.30 & 1.10 & 14.8 & 22.4 & NS \\
\hline
\end{tabular}

*Measurements in mm; Number of the sample: 20 males and 25 females.

SD: Standard deviation, NS: Not significant $(p>0.05)$.

Table (4) illustrated the description of linear canine distances for the total samples with their comparison. The mean values were found nearly equal and always the distal records were found to be larger than the mesial and the post treatments larger than the pre treatment measurements, alth- ough the minimum values for pre was less than the post, while in maximum record, the pre treatment measurements were found to be larger than the post treatment measurements. These variability may be related to that different age groups were taken together as one group.

Table (4): Description of linear canine distances pre and post treatment with their comparison of total value

\begin{tabular}{ccccccc}
\hline Side & Treatment & Mean* & \pm SD & Minimum & Maximum & $\boldsymbol{p}$-value \\
\hline \multirow{2}{*}{ Mesial } & Pre & 13.85 & 1.12 & 11.6 & 16.5 & 0.66 \\
& Post & 13.99 & 1.00 & 12.0 & 15.7 & NS. \\
\hline \multirow{2}{*}{ Distal } & Pre & 18.50 & 1.80 & 12.4 & 22.0 & 0.9 \\
& Post & 18.54 & 1.10 & 17.0 & 21.0 & NS. \\
\hline
\end{tabular}

Table (5) showed the description of linear post-pre treatment measurements according to age group, which represent post minus pre treatment records for both mesial and distal distances. Positive $(+\mathrm{ve})$ and negative $(-\mathrm{ve})$ records could be seen. Positive values were considered normal because those records yield from post distance, which was already larger than the pre distance because of canine position in the post treatment located more posteriorly than its position in the pre treatment case. While -ve values mean that the pre distance was larger than the post and this opposi- 
te to the normal. These gave information about rotation in the -ve value side toward the median palatal raphe (Figure $4 \mathrm{n}$ ).

The majority of the mean values were -ve, these mean smaller post than pre distances and so there was constriction in the arch width mainly in canine position. Also, for the second and fourth groups, the mean value for the mesial was larger than the distal. Generally, the distal distance was larger than the mesial for the canine because of its position at the angle of the mouth be- tween anterior and posterior segments, while, in the result of this study, in second group the opposite was found. It means canine rotation in a way that distal side rotate toward the median palatal raphe. In the fourth group, the mesial and distal values were +ve. So, there was no constriction in the dental arch but still there was rotation in the mesial side out of the dental arch (Figure $4 \mathrm{~m}$ ). These differences of the fourth group from the other three groups may be related to the age.

Table (5): Description of linear post-pre

measurements according to age group

\begin{tabular}{cccccc}
\hline \multirow{2}{*}{ Groups } & \multicolumn{2}{c}{ Mesial } & \multicolumn{2}{c}{ Distal } & \\
\cline { 2 - 5 } & Mean* & \pm SD & Mean* & \pm SD & -value \\
\hline \multirow{2}{*}{ First } & -0.27 & 1.00 & 0.10 & 2.55 & $\begin{array}{c}0.84 \\
\text { NS }\end{array}$ \\
Second & 0.27 & 0.65 & -0.97 & 0.60 & $\begin{array}{c}0.10 \\
\text { NS }\end{array}$ \\
Third & -1.07 & 1.68 & -0.32 & 3.85 & $\begin{array}{c}0.68 \\
\text { NS }\end{array}$ \\
& & & & & 0.29 \\
Fourth & 1.73 & 1.30 & 0.85 & 0.50 & NS \\
\hline
\end{tabular}

*Measurements in mm

SD: Standard deviation, NS: Not significant $(p>0.05)$.

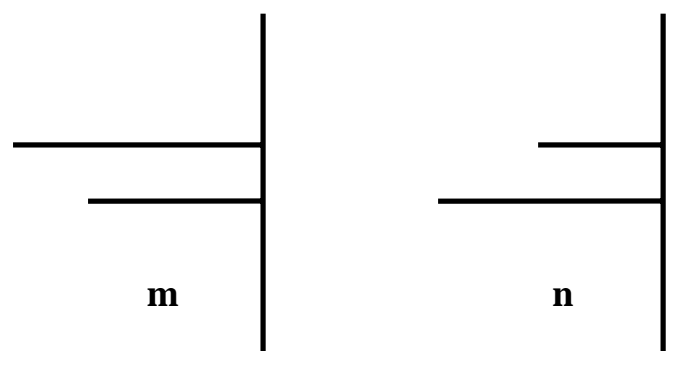

In the first and third groups, the distal distance was found to be larger than the mesial. This means that there was no rotation, although a -ve value was seen for both mesial and distal distances, which means constriction of canine distance from median palatal raphe; while in the first group the mesial distance was -ve. So, there was rotation of mesial side toward the palatal raphe. This may be due to controlling treatment method for all the patients and using the same materials (arch wire and ligatures) which were stainless steel and tight ligation between ligatures and arch wire. While the slight rotation which occurred might be related to the flexibility of sta-
Figure (4): Canine rotation

m: Rotation out of the dental arch (away from reference line.

$\mathrm{n}$ : Rotation toward the reference line.

inless steel arch wire and ligatures and these agreed with the results of several studies, ${ }^{(11,17-19)}$ which proved that the ligature could contact the wire at one or both mesiolabial and distolabial with the bracket and if tight ligation there was no rotation allowed during tooth retraction.

Matasa $^{(14)}$ and Daniel ${ }^{(20)}$ used different bracket designs and they found an increase tendency toward distal rotation or buccal root torque were recorded, because those brackets giving the arch wire increase in mobility within them or lack of ligature wire. Other researches ${ }^{(20,21)}$ related the rotation was that the center of resistance (rotation) theoretically located on the long ax- 
is of the tooth, but the point of force application was buccal or labial to the long axis or the teeth usually rotate out to the labial to a greater arch radius (if the arch wire larger than the dental arch).

\section{Angular Measurements}

The angular measurements were the familiar method for measuring canine rotation as measured by Ziegler and Ingervall. ${ }^{(9)}$

Table (6) showed the comparison of angular canine rotation for each side (total sample). A non-significant difference between right and left sides was found. The same result was found for the linear measurements in Tables (2) and (3).

Table (7) showed the descriptive of the variables (pre and post canine rotation) for the total sample with their comparison. Also, it can be noted that the mean values were nearly equal for that a non-significant difference was found between pre and post treatment angular records. The same findings in the linear measurements were seen in Table (4).

Table (8) demonstrated the descriptive of angular canine rotation (pre and post) according to age group with their comparison. In the second and fourth groups the pre treatment records were larger than the post records and these mean there was rotation in the canine, while in the first and third groups the post treatment records found to be larger than the pre records. The differences between pre and post records angles were not significant. This result coincided with the linear records in Table (5). From Table (8), the side of rotation whether in mesial or distal side could not be noted.

Table (6): Comparison of angular canine rotation for each side

\begin{tabular}{ccccc}
\hline Treatment & Side & Mean* & \pm SD & $\boldsymbol{p}$-value \\
\hline \multirow{2}{*}{ Pre } & Right & 33.45 & 6.04 & 0.6 \\
& Left & 31.53 & 7.12 & NS \\
\hline \multirow{2}{*}{ Post } & Right & 30.82 & 6.92 & 0.18 \\
& Left & 32.10 & 7.65 & NS \\
\hline
\end{tabular}

*Measurements in mm

SD: Standard deviation, NS: Not significant ( $p>0.05)$.

Table (7): Comparison between pre and post treatment for canine rotation

\begin{tabular}{cccccc}
\hline Treatment & Mean* $^{*}$ & $\mathbf{\pm}$ SD & Minimum & Maximum & $\boldsymbol{p}$-value \\
\hline Pre & 32.49 & 6.52 & 20.0 & 44.5 & 0.84 \\
Post & 32.92 & 7.40 & 16.0 & 45.0 & NS \\
\hline
\end{tabular}

*Measurements in mm; Number of the sample $=45$.

SD: Standard deviation, NS: Not significant $(p>0.05)$.

Table (8): Description of angular canine rotation (post-pre) according to age group

\begin{tabular}{ccccc}
\hline Groups & Treatment & Mean* & \pm SD & $\boldsymbol{p}$-value \\
\hline \multirow{2}{*}{ First } & Pre & 31.90 & 4.82 & 0.06 \\
& Post & 37.10 & 4.34 & NS \\
\multirow{2}{*}{ Second } & Pre & 32.15 & 8.15 & 0.16 \\
& Post & 30.03 & 8.05 & NS \\
Third & Pre & 31.75 & 5.84 & 0.66 \\
& Post & 31.86 & 3.55 & NS \\
\multirow{2}{*}{ Fourth } & Pre & 36.63 & 7.89 & 0.56 \\
& Post & 34.38 & 4.53 & NS \\
\hline
\end{tabular}

*measurements in degree

SD: Standard deviation, NS: Not significant $(p>0.05)$. 
From the two methods of measuring canine rotation (linear and angular), in the work the linear measurements was directly from the study cast of the patient (pre and post models) by using vernia. The accuracy of this device to $0.1 \mathrm{~mm}$ and the measurements directly from the mesial and distal contact points of the canine vertical to the palatal raphe (pre and post). So, four measurements could be obtained, then by subtracting post minus pre, the amount and side of canine rotation could be gotten.

In the second method (angular), the measurements must be converted which were mesial, distal and canine width to the paper then drawing the continuation line of canine width with the line of palatal raphe to form angle in which the difference between pre and post values gave the amount of canine rotation which was measured by protector and here the accuracy to $0.5 \mathrm{~mm}$ degree. So, eight measurements could be obtained: The pre linear measurements were three (mesial, distal and canine width) and one angular done on a paper, then four post measurements which also recorded on a paper.

\section{CONCLUSION}

Canine rotation occurred within the treatment work in the two methods of measuring canine rotation, but this research proved that the linear direct measurement which is the new method followed in this work was more beneficial and accurate for clinical work than the indirect angular measurements and gave more information about the position of the canine in post treatment.

\section{REFERENCES}

1. Bourauel C, Freuden Reich D, Vollmer D, Kobe D, Drescher D, Jagar A. Simulation of orthodontic tooth movement: A comparison of numerical models. J Orofac Orthop. 1999; 60(2): 136-151.

2. Kojima Y, Fukui H. Numerical simulation of canine retraction by sliding mechanics. Am $J$ Orthod Dentofac Orthop. 2005; 127(5): 542-551.

3. Eric JW, Shing Huany D. Rapid canine retraction through destruction of the periodontal ligament. Am J Orthod Dentofac Orthop. 1998; 114: 372-382.
4. Yijin Ren D, Jaap C, Anne M. Optimum force magnitude for orthodontic tooth movements. Angle Orthod. 2002; 72(1): $68-92$.

5. Perez CA, Caputo A, Chaconas SJ. Canine retraction with J-hook headgaar. Am J Orthod. 1980; 78: 538-547.

6. Jessing G. Biomechanical design and clinical evaluation of new canine retraction spring. Am J Orthod. 1985; (88)9: 88-93.

7. Bia D, Cheng BH, Luo SJ, Lu T. Three dimensional finite element analysis of maxillary canine during translation movement. Sichuan Da Xue Bao. 2004; 35(3): 358-360 (English Abstr).

8. Rudolph DJ, Willes PMC, Sameshine GI. A finite element model of apical force distribution from orthodontic tooth movement. Angle Orthod. 2001; 71(2): 127-131.

9. Ziegler E, Ingervall F. Maxillary canine retraction with retraction spring and sliding mechanics. Am J Orthod Dentofac Orthop. 1989; 95: 99-106.

10. Grekmore G, Kunk B. Straight wire: The next generation. Am J Orthod Dentofac Orthop. 1993; 104(8): 18-20.

11. Bednar A, Gruendeman C. Influence of bracket design on movement production during rotation. Am $J$ Orthod Dentofac Orthop. 1993; 104(9): 254-261.

12. Staggers JA, German N. Clinical consideration in the use of retraction mechanics. Am J Orthod Dentofac Orthop. 1991; 101(3): 364-369.

13. Philippe J. A system for retracting canine roots. Am J Orthod Dentofac Orthop. 1987; 91(4): 400-404.

14. Matasa CG. Bracket angulation as a function of its length in canine distal movement. Am J Orthod Dentofac Orthop. 1997; 111(2): 178-184.

15. Schumacher HA, Bourawel C, Dreschar D. The influence of bracket design on frictional losses in the bracket/archwire system. J Orofac Orthop. 1999; 60(5): 335-347.

16. Omana HM, Moore RN, Bagb MD. Frictional properties of metal and ceramic brackets. Am J Orthod Dentofac Orthop. 1992; 102(9): 177-182.

17. Lu Wang, Tarng C. Force decay of elastomeric chain. Am J Orthod Dentofac Orthop. 1993; 104(10): 373-377.

18. Pieter W, Van Heerden M, Jozue P. Der- 
otating a tooth with lingual rotation tie. $J$ Clin Orthod. 1991; 8: 160-162.

19. Frank A. Frictional resistance between orthodontic bracket and archwire. Am J Orthod. 1980; 78(12): 593-609.

20. Daniel JJ. A technique to reduce archwire binding. J Clin Orthod. 1996; 13: 150153.
21. Yamaguchi Z. A study of force application, amount of retarding force and bracket width in sliding mechanics. Am J Orthod Dentofac Orthop. 1996; 109(1): 5056.

22. David M. Clinical aid avoiding cross-contamination of elastomeric ligatures. $J$ Clin Orthod. 1993; 10: 538-538. 